\title{
Multidisciplinary Team Meetings for Patients with Complex Extremity Defects - A Retrospective Analysis of Treatment Recommendations and Prognostic Factors for Non-Implementation.
}

\section{Dimitra Kotsougiani-Fischer ( $\nabla$ dkotsougiani@googlemail.com )}

Berufsgenossenschaftliche Unfallklinik Ludwigshafen

\section{Sebastian Fischer}

Berufsgenossenschaftliche Unfallklinik Ludwigshafen

Jan Warszawski

Berufsgenossenschaftliche Unfallklinik Ludwigshafen

\section{Paul Gruetzner}

Berufsgenossenschaftliche Unfallklinik Ludwigshafen

\section{Gregor Reiter}

Berufsgenossenschaftliche Unfallklinik Ludwigshafen

Christoph Hirche

Berufsgenossenschaftliche Unfallklinik Ludwigshafen

\section{Ulrich Kneser}

Berufsgenossenschaftliche Unfallklinik Ludwigshafen

\section{Research Article}

Keywords: multidisciplinary team meetings, complex extremity defects, free flap, bone reconstruction

Posted Date: December 28th, 2020

DOl: https://doi.org/10.21203/rs.3.rs-125873/v1

License: (9) This work is licensed under a Creative Commons Attribution 4.0 International License. Read Full License

Version of Record: A version of this preprint was published at BMC Surgery on March 29th, 2021. See the published version at https://doi.org/10.1186/s12893-021-01169-4. 


\section{Abstract}

\section{BACKGROUND}

This study aimed to assess a multidisciplinary team (MDT) meeting approach for the management of patients with complex extremity defects, analyze treatment recommendations, and evaluate factors influencing non-implementation.

\section{METHODS}

All patients introduced to an MDT meeting for complex extremity defects from 2015 to 2017 were included. Patients' characteristics and defect causes were evaluated. Treatment recommendations of MDT meetings and subsequent implementation were reviewed, and factors associated with nonadherence to recommendations were statistically analyzed using logistic regression.

\section{$\underline{\text { RESULTS }}$}

Fifty-one patients (41 male) with a mean age of 54 years were presented in 27 MDT meetings. Most of the patients $(70 \%)$ suffered from reconstructive challenging or combined bone- and soft tissue defects, primarily located at the lower extremity (88\%). Large skeletal defects, chronic osteomyelitis, and multifragmented fractures were present in $65 \%$ of cases. Forty-five percent of the patients suffered from peripheral vascular disease, necessitating surgical optimization. Of the 51 MDT decisions, 40 were implemented (78\%; (32/40) limb salvage versus 22\%; (8/40) limb amputation). Limb salvage was successfully achieved in $91 \%(29 / 32)$ of the cases. Failed limb salvages were due to flap failure (33\%; $1 / 3)$, recurring periprosthetic joint infections $(66 \% ; 2 / 3)$ and concomitant reconstructive failure. Patients who underwent limb amputation, as recommended, showed proper stump healing and regained mobility with a prosthesis. Overall the MDT treatment plan was effective in $92.5 \%(37 / 40)$ of the patients, who adhered to the MDT treatment recommendation. In eleven patients $(22 \% ; 11 / 51)$, the MDT treatment was not implemented. MDT decisions were less likely to be implemented, if amputation was recommended ( $p$ $=0.029$.

\section{CONCLUSIONS}

MDT meetings represent a valid tool to formulate individualized treatment plans, avoiding limb amputation in most patients with severe extremity defects. Recommendation for limb amputation is less likely to be implemented than plans for limb salvage.

\section{TRIAL REGISTRATION:}

Retrospectively registered

\section{Background}


In the past decades, multidisciplinary and innovative therapeutic concepts have revolutionized the treatment of complex extremity defects, thus successfully averting limb amputation in many cases. Depending on the defect size and localization, involvement of tissue components, and presence of infection, the reconstructive approach can range from simple to complex procedures. Large osseous defects may necessitate cryopreserved allogeneic or microvascular autologous bone transplants, bone prostheses, or bone transport via Masquelet technique [1-3]. Concomitant or extensive soft tissue defects may require microvascular free flap coverage and severe damage to muscle groups may indicate complex tendon transfer or muscle neurotization $[4,5][6]$

In addition, depending on the underlying disease, optimization of the vascular status might be necessary. Finally, patient-related factors such as comorbidities, patient preferences, and functional status influence the treatment plan, making a multidisciplinary team approach mandatory in the successful treatment of complex extremity defects $[7,8]$.

Multidisciplinary team (MDT) meetings are well-established for treatment recommendations in patients with cancer. They serve as a platform to provide expert reviews on patient cases, according to clinical practice guidelines and beyond, ensuring well-coordinated and multi-professional patient care [9]. Similarly, regularly scheduled MDT meetings, comprising professionals from trauma and orthopedic, plastic-, and vascular surgery, can formulate individualized treatment recommendations for patients with complex extremity defects. Thus, in 2015, an MDT meeting for patients with complex extremity defects the so-called extremity board - was established in our unit. However, MDT meetings require significant time resources and finances. Thus, it is reasonable to question their impact on patient outcomes and evaluate their quality. An excellent method to analyze MDT performance is to assess implementation of the formulated treatment plan along with the clinical outcome. Therefore, this study aimed to investigate the implementation of treatment decisions reached in the MDT meeting for patients with complex extremity defects and determine factors associated with non-implementation of the recommendations. The secondary aim was to evaluate all implemented board decisions' clinical outcome as a criterion for the quality of the reached multidisciplinary treatment decision.

\section{Patients And Methods}

\section{MDT meetings for patients with complex extremity defects}

MDT meetings were held monthly at the facility of the senior author. Meetings were certified for continuous medical education credit points and announced biannually in print and via email to physicians of surrounding hospitals and private practice. The MDT consisted of trauma, orthopedic, plastic and vascular surgeons. Professionals of other disciplines (rehabilitation therapy, radiology, critical care, geriatrics) were invited if indicated. The treating physician submitted cases for presentation to the coordinator of the MDT meeting (first author DK) for review and acceptance. Submissions included a check-list that ensured medical history, relevant images ( $\mathrm{x}$-ray, computed tomography, magnetic resonance imaging, angiography), clinical findings and microbiological results were avaialble [10]. 
Registration of cases ended two days before the next meeting to provide sufficient time for acquiring further documents or invitation of professionals of other disciplines. In the case of vascular pathologies, all relevant angiographies were transferred to a vascular surgeon before the upcoming meeting with means of teleradiology. During the MDT meeting, patient cases were presented summarizing all relevant clinical data and demonstrating all relevant imaging. After the MDT meeting, the reached treatment decision was documented in the electronic patient file. The treating physician informed the patient of the treatment recommendation. Figure 1 displays the workflow of the MDT meetings for patients with complex extremity defects.

\section{Study design}

The local ethics committee of Rhineland palatinate (no. 2020-15004) Mainz Germany approved the retrospective study, which was designed in accordance with the ethical standards laid down in the Declaration of Helsinki and its later amendments. Since the study protocol included only a retrospective, epidemiologic evaluation of anonymized and routine patient demographics, procedural and outcome specific parameters informed consent was waived by the local ethics commitee of Rhineland palatinate.

All medical records from patient cases presented in the MDT meetings for complex extremity defects between September 2015 and December 2017 were retrospectively studied. Epidemiologic data, such as age, gender, comorbidities, American Society of Anesthesiologists (ASA) physical status classification system, defect localization, extent of defect (bone, soft tissue, combined bone and soft tissue defect) and vascular status at the time of the MDT meeting were identified. Furthermore, MDT treatment decision and implementation were evaluated and classified as implemented (MDT treatment decision and treatment received were the same) or as non-implemented (MDT decision differed from final MDT treatment). Furthermore, reasons for non-implementation of MDT treatment decisions were gathered (patient's preference, comorbid conditions, or new clinical information). Last, but not least, the clinical course of all patients who adhered to the MDT treatment recommendation was analyzed.

\section{Statistical methods}

Data are presented as frequencies (percentages) for the categorical variables and means - standard deviation (SD) for the continuous variables. A multivariable logistic regression model was performed to identify factors associated with the non-implementation of MDT treatment decisions. Hosmer/Lemeshow $(\mathrm{HL})$ tests for calibration were computed to assess the goodness of fit. The odds ratios (OR) with their corresponding 95\% confidence intervals (Cls) were calculated.. Statistical significance was defined as $p<$ 0.05. Data were analyzed using the GraphPad Prism version 8.4.0 for MAC (GraphPad Software San Diego, CA).

\section{Results}

Demographic data 
During the study period, 27 MDT meetings were held, in which 51 patients with complex extremity defects were presented. Representatives of trauma surgery and orthopedics, as well as plastic surgery, were present in all MDT meetings. Vascular surgeons were present in $19.6 \%(10 / 51)$ of cases. In the remaining $80.4 \%(41 / 51)$ of the cases, telemedicine consultations were performed with vascular surgeons. Forty-one patients were male and ten female. The mean age was 54 years, ranging from 21 to 81 years. Twenty percent of the presented patients were active smokers and $43.1 \%$ suffered from hypertension.

Furthermore, diabetes mellitus was present in $23.5 \%$ of the patients.

The majority of patients had lower limb defects $(88.2 \%, 45 / 51)$. Upper extremity defects were less common $(11.8 \%, 6 / 51)$. Thirty-six patients $(70.6 \%, 36 / 51)$ had combined bone and soft tissue defects. Isolated soft tissue defects occurred in $22 \%$ of cases $(11 / 51)$ and isolated bone defects in $8 \%$ of cases $(4 / 51)$. Of the 40 patients with bone defects (combined + isolated), segmental bone defects (defect size > $2 \mathrm{~cm}$ ) were present in $27.5 \%$ of patients (11/40), complex multi-fragmented fractures in $27.5 \%(11 / 40)$ and osteomyelitis in $27.5 \%(11 / 40)$. Furthermore, periprosthetic joint infections were apparent in three patients $(5.9 \%)$. The majority of defects were due to acute trauma $(56.9 \%, 29 / 51)$. Rarely, peripheral vascular disease $(7.8 \%, 4 / 51)$, tumors $(3.9 \%, 2 / 51)$, or infection $(30 \%, 1 / 51)$ were responsible for the extremity defect. Almost one third of patients $(27.5 \%, 14 / 51)$ had previously received a peripheral vascular surgery and $17.6 \%(9 / 51)$ had pathologies in angiography at the time of the MDT meeting. Epidemiologic data are presented in Table 1. 
Table 1

Epidemiologic data

\begin{tabular}{|c|c|}
\hline Variables & Patients $\mathrm{n}=\mathbf{5 1}$ \\
\hline Age, M (SD), years & $53.9 \pm 17.2$ \\
\hline Gender, male, N (\%) & $41(80.4 \%)$ \\
\hline ASA physical health status, M (SD) & $2.2 \pm 0.8$ \\
\hline Smoking history, N (\%) & $10(19.6 \%)$ \\
\hline Hypertension, N (\%) & $22(43.1 \%)$ \\
\hline Diabetes mellitus, N (\%) & $12(23.5 \%)$ \\
\hline \multicolumn{2}{|l|}{ Defect localization } \\
\hline Upper extremity, N (\%) & $6(11.8 \%)$ \\
\hline Lower extremity, N (\%) & $45(88.2 \%)$ \\
\hline \multicolumn{2}{|l|}{ Extent of defect } \\
\hline Isolated bone, N (\%) & $8(4 \%)$ \\
\hline Isolated soft tissue, N (\%) & $22(11 \%)$ \\
\hline Combined bone and soft tissue, N (\%) & $36(70.6 \%)$ \\
\hline \multicolumn{2}{|l|}{ Classification of bone defects } \\
\hline \multicolumn{2}{|l|}{ (defect size > $2 \mathrm{~cm}), \mathrm{N}(\%)$} \\
\hline Complex multi-fragmented fractures, $\mathrm{N}(\%)$ & $11(21.6 \%)$ \\
\hline Osteomyelitis, N (\%) & $11(21.6 \%)$ \\
\hline Periprosthetic joint infection, N (\%) & $3(5.9 \%)$ \\
\hline \multicolumn{2}{|l|}{ Defect cause } \\
\hline Peripheral vascular disease, N (\%) & $4(7.8 \%)$ \\
\hline Tumors, N (\%) & $2(3.9 \%)$ \\
\hline Infection, N (\%) & $16(31.4 \%)$ \\
\hline Acute trauma, $\mathrm{N}(\%)$ & $29(56.9 \%)$ \\
\hline Previous vascular surgery, N (\%) & $14(27.5 \%)$ \\
\hline Pathologic vascular status at MDT meeting, $\mathrm{N}(\%)$ & $9(17.6 \%)$ \\
\hline
\end{tabular}




\section{Outcomes}

The follow-up amounted to $47.1 \pm 8.6$. Agreement in MDT treatment decisions was reached in all presented cases. Limb salvage was recommended for $37 / 51$ (72.5\%) patients, and limb amputation for $14 / 51(27.5 \%)$ patients.

In over half of the patients (56.9\%), a plastic surgery intervention was recommended, most commonly with free flaps (51\%). When free flaps were necessary to close the defect, complex chimeric flaps were predominantly recommended, such as the combined parascapular and latissimus dorsi free flap. In over $20 \%$ of the patients, a vascular intervention or additional radiologic analyses were proposed. Furthermore, in $39.2 \%$ of patients, complex bone reconstructive options were recommended. These included avascular and vascularized bone grafting and staged procedures, such as antibiotic spacer implantation and distraction osteogenesis. MDT treatment decisions for limb reconstruction are presented in detail in Table 2. 
Table 2

MDT treatment recommendations for limb preservation

\begin{tabular}{|ll|}
\hline Variables & Patients n = 51 \\
\hline Vascular surgery intervention & $11(21.6 \%)$ \\
\hline Arteriovenous loop & $2(3.9 \%)$ \\
\hline Percutaneous transluminal angioplasty & $1(2 \%)$ \\
\hline Angiography & $4(7.8 \%)$ \\
\hline Vessel-extension by vein graft & $2(3.9 \%)$ \\
\hline Plastic Surgery intervention & $29(56.9 \%)$ \\
\hline Free flaps & $26(51.0 \%)$ \\
\hline Chimeric parascapular and latissimus flap & $8(15.7 \%)$ \\
\hline Free groin flap & $1(2.0 \%)$ \\
\hline Free iliac crest flap & $1(2.0 \%)$ \\
\hline Latissimus dorsi flap & $7(13.7 \%)$ \\
\hline Anterior lateral thigh flap & $4(7.8 \%)$ \\
\hline Parascapular flap & $2(3.9 \%)$ \\
\hline Rectus abdominis flap & $1(2.0 \%)$ \\
\hline Free fibular flap & $2(3.9 \%)$ \\
\hline Pedicled flap & $3(5.9 \%)$ \\
\hline Trauma Surgery intervention & $20(39.2 \%)$ \\
\hline Osteosynthesis & $10(19.6 \%)$ \\
\hline Antibiotic spacer removal & $1(2.0 \%)$ \\
\hline Antibiotic spacer implantation & $3(5.9 \%)$ \\
\hline Bone debridement & $7(13.7 \%)$ \\
\hline Arthrodesis & $4(13.7 \%)$ \\
\hline Endoprosthesis & $1(2.0 \%)$ \\
\hline Resection arthroplasty & $3.9 \%)$ \\
\hline Bioactive glass scaffold implantation & \\
\hline Autogenous Bone grafting & \\
\hline MDT, multidisciplinary team; & \\
\hline
\end{tabular}




\begin{tabular}{|ll|}
\hline Variables & Patients $\mathbf{n = 5 1}$ \\
\hline Allogeneic Bone grafting & $1(2 \%)$ \\
\hline Implant removal & $7(13.7 \%)$ \\
\hline Other intervention & $\mathbf{5 ( 9 . 8 \% )}$ \\
\hline Intraoperative demonstration & $1(2 \%)$ \\
\hline Home vacuum therapy & $4(7.8 \%)$ \\
\hline MDT, multidisciplinary team; & \\
\hline
\end{tabular}

Overall, $78.4 \%$ (40/51) of the MDT treatment decisions were implemented. In the remaining cases $(21.6 \%$; $11 / 51$ ), in which MDT treatment decision was not implemented, the main reason for discordance was the patient's preference $(6 / 51,11.8 \%)$; patients chose limb salvage or no treatment instead of the recommended lower leg amputation. Further reasons for non-implementation of MDT recommendations included comorbidities $(2 / 51,3.9 \%)$ and new clinical information $(3 / 51,5.9 \%)$, which were not available at the time of MDT meeting and made limb salvage impossible (Table 3 and Table 4).

Table 3

Reasons for non-implementation of MDT treatment decisions

\begin{tabular}{|ll|}
\hline Variables & Patients $\mathbf{n = 5 1}$ \\
\hline Patient's preference, N (\%) & $6(11.8 \%)$ \\
\hline Comorbidities, N (\%) & $2(3.9 \%)$ \\
\hline New clinical information, N (\%) & $3(5.9 \%)$ \\
\hline M, mean; SD, standard deviation. \\
\hline
\end{tabular}


Table 4

Conversion of MDT treatment recommendations

\section{Reason for non- implementation \\ MDT treatment recommendation \\ Final course of action}

Implant removal ankle, free flap

reconstruction lower leg
Implant removal, conservative wound treatment, shock wave therapy

Conservative wound treatment, negative pressure therapy

preference

Lower leg amputation

Lower leg amputation

Patient's

preference

Patient's

preference

Patient's Free flap reconstruction, ankle arthrodesis

preference

with bone grafting

Patient's Lower leg amputation

preference

Comorbidity

(severe PVD)

AV-Loop, free flap reconstruction lower leg

Vascular imaging, Free flap reconstruction,

lower limb amputation

Comorbidity
(severe PVD)

Humerus reconstruction by fibular free flap reconstruction, plate osteosynthesis, radial

New clinical

information

(insufficient

perfused soft

tissue and

muscles) artery reconstruction, fasciocutaneous free

flap reconstruction, median nerve

reconstruction through sural nerve grafting

New clinical

information

(new inguinal

wound healing

problem)

Femur-removal, lower leg amputation, reconstruction with osteomyocutaneous turnup plasty as described by Sauerbruch

Comorbidity (severe PVD)

Vascular imaging, Free flap reconstruction, lower limb amputation

New clinical information (soft tissue of forefoot viable)
Conservative wound treatment

Ilizarov bone transport

Free flap reconstruction, simple osteosynthesis

Conservative wound treatment, negative pressure therapy

Iliac artery angioplasty, femoral artery angioplasty,

PTA A. poplitea, lower leg amputation, prosthesis

Transfemoral amputation

Upper limb amputation, prosthesis
Forefoot amputation, chimeric free flap reconstruction of foot
Femur-removal, lower leg amputation, reconstruction with osteomyocutaneous turn-up plasty as described by Sauerbruch, pedicled flap reconstruction

Transfemoral amputation

Osteosynthesis, free flap reconstruction of the feet, skin grafting

Multidisciplinary team, MDT; peripheral vascular disease, PVD; percutaneous transluminal angioplasty, PTA; 
Multiple logistic regression analysis exploring factors that might influence the implementation of the MDT meeting decision showed that amputation intent was an important reason not to follow the recommendation with 6-fold greater odds $(\mathrm{Cl} 1.4-29.4 ; \mathrm{p}=0.021)$. Gender, age, and physical status (ASAclassification) did not influence the implementation of MDT treatment decisions (Table 5).

Table 5

Prognostic risk factors for non-implementation of MDT treatment decision

\begin{tabular}{|lccc|}
\hline Variables & Odds ratio & $(95 \% \mathrm{Cl})$ & $p$-value \\
\hline Gender & 0.2 & $(0.1-2.1)$ & 0.234 \\
\hline Female & & & \\
\hline Age & 0.6 & $(0.1-3.1)$ & 0.530 \\
$\geq 65$ & & $(0.3-7.6)$ & 0.656 \\
\hline ASA-classification & 1.5 & & \\
\hline ASA 3-4 & & $(1.4-29.4)$ & $0.021 *$ \\
\hline Treatment intent & 6.0 & & \\
\hline Amputation & & & \\
\hline Multidisciplinary team, MDT; American Society of Anaesthesiologists, ASA; * significant, $p<0.05$. \\
\hline
\end{tabular}

To evaluate the quality of the MDT treatment recommendations, the final clinical outcome of all patients, who adhered to the MDT treatment recommendation (78.4\%; 40/51), was followed. Limb salvage was recommended for $80 \%$ (32/40) of the patients and successfully achieved in $90.6 \%$ (29/32) of the cases. Failed limb salvages were due to flap failure $(33 \% ; 1 / 3)$, recurring periprosthetic joint infections $(66 \%$; $2 / 3$ ), and concomitant reconstructive failure. Of note, in all patients with failed limb salvage, vascular interventions had been performed (one patient with arteriovenous loop and two patients with vessel extensions by vein grafts). All three patients with failed extremity reconstructions were debriefed in the following MDT meeting for extremity defects. Patients with amputations $(20 \% ; 8 / 40)$ showed proper stump healing and regained mobility with a prosthesis. Overall, the MDT treatment plan was effective in $92.5 \%(37 / 40)$ of the patients, who adhered to the MDT treatment recommendation.

\section{Discussion}

This consecutive series of treatment recommendations made within an MDT meeting for patients with complex extremity defects found that the formulated treatment plan was effective in $92.5 \%$ of the patients who adhered to the MDT treatment recommendation. Limb salvage was recommended with a complex reconstructive procedure for $72.5 \%$ of the patients and limb amputation in $27.5 \%$ of the cases. However, in $22 \%$ of the cases the MDT treatment decision was not implemented. Of the decisions that changed after the meeting, the most common reason was that it was unacceptable to the patient. In 
particular, the decision to amputate an extremity was significantly associated with the nonimplementation of the MDT treatment plan.

MDT meetings are nowadays mandatory for treatment decisions in patients with malignancies. Interestingly, recent studies show that a discordance from the initial tumor board decision in patients with breast cancer is found in $8 \%$ of cases [11]. In patients with colorectal or gastrointestinal tumor, the therapy can differ in as much as $10-40 \%$ cases from the initial tumor board decision [12]. It should be borne in mind that there are better data and evidence in oncology and well-developed treatment guidelines. In contrast, no specific guidelines exist for the treatment of devastating or composite softtissue and bone defect. Each case is unique and necessitates an individualized therapeutic approach. We detected a moderate rate of discordance (21.9\%) between the initial MDT treatment decision and final treatment plan. The key reason for non-implementation of MDT recommendations in $11.8 \%$ of the cases was that the MDT treatment decision was not acceptable to the patient. Final treatments were more conservative than initially planned, choosing no therapy or limb reconstruction instead of amputation. In oncology, the patient's preference is one of the most important factors for a discordance between the treatment decision of the MDT meeting and final clinical outcome [13]. An option to increase patient's adherence to the MDT treatment plan may be the involvement of patients in the treatment decision process by including them in the MDT meeting. This topic has caused controversy in several previous studies focusing on multidisciplinary breast cancer conferences [14, 15]. However, research on the risks and benefits of patient participation has not provided substantiated findings yet [14]. Furthermore, Hamilton et al. showed recently that MDT decision-making process needs a substantial review if patients are to be effectively involved [16].

Further reasons for non-implementation of treatment plans were comorbidities (3.9\%) and new clinical information (5.9\%), which made it impossible to implement the board's decision, including periprocedural risk factors. This is an important finding, which has to be addressed in order to improve the quality of the MDT extremity board. However, the lack of consideration of the patient's comorbidities during multidisciplinary tumor conference decision-making has also been demonstrated in previous studies [13]. One strategy to improve MDT meetings' structural quality is to use checklists for patient case preparations [17]. Although our MDT meeting checklist tool did include a section for patient's comorbidities, new clinical information or worsening of a clinical diagnosis may inhibit the implementation of a treatment decision.

This study also identified factors associated with the non-implementation of the MDT treatment plan. As expected, the MDT treatment decision for limb-amputation was a significant factor in not adhering to the treatment decision $(p=0.029)$. Patients preferred to get no therapy or insisted on the attempt of complex reconstructive procedures in the view of a rather low chance of success instead of limb amputation. However, postoperative functionality and survival benefit were critical factors in the decision-making process $[18,19]$. Thorough patient education is urgently needed to increase therapy adherence. Furthermore, debriefing of changed therapeutic plans is necessary when the MDT treatment decision is not followed. 
The concept of MDT meetings for patients with complex extremity defects is still relatively new [10]. In 2015 we established these MDT meetings comprising experts from orthopedic and trauma, plastic and vascular surgery analogous to multidisciplinary tumor board meetings. The aim was to find a treatment plan for challenging cases, in which patients presented with complex defects of the extremities, involving at least two surgical disciplines for limb salvage (Fig. 2). Furthermore, patients with limb-threatening diseases in whom the feasibility and sense of limb preservation was insecure were presented. Of note, approximately 300 free flap extremity reconstructions are performed annually in our clinic, of which only 5-6\% were presented in the MDT meeting for extremity defects.

The complexity and severity of the cases presented in the MDT meeting are further reflected by the high number of patients with combined bone- and soft tissue defects (70\%) as well as extensive skeletal defects, multi-fragmented fractures, or infected bone (64.7\%). Furthermore, $45.1 \%$ of the patients had a pathologic vascular status, complicating the therapy.

Although $70.6 \%$ of the patients from this study suffered from extensive, combined bone and soft tissue defects, the decision to restore the extremity was reached in almost two-thirds of the patients. When the MDT treatment plan for limb reconstruction was followed, limb salvage with proper weight-bearing was achieved in $91 \%$ of cases. This high percentage of weight-bearing patients is quite satisfying, taking into consideration the severity of limb-threatening diseases. In patients, in which limb amputation was the only viable option, the MDT meeting provided a platform to define the optimal limb length. Our outcome analysis showed that patients with recommended limb amputation showed a proper stump healing and regained the ability to walk with prosthesis. Telemedicine was used in quite a high percentage of patients $(80 \%)$ to ensure all participating disciplines' input and presence for effective decision-making. In this context, a recent study from Endean et al. showed that telemedicine evaluation of patients with vascular pathologies is accurate and effective, and compares to on-site evaluations [20].

The requirements for a functioning MDT meeting for patients with complex extremity defects were comparable to those of cancer MDT meetings $[13,17]$. The MDT meeting had a sensible team structure and was managed by an integratively-acting expert in extremity reconstruction. Structured presentations were adapted to standardized checklists, including complete diagnosis, as well as patient-related factors such as psychosocial factors and patient preferences regarding the treatment recommendation. For the MDT treatment decision, an expert committee consisting of decision-makers from all surgical disciplines was present. Auditing of non-implementation of MDT treatment decisions and studying reasons for changed decisions provided us useful feedback. To maximize MDT meeting performance and to achieve patient-centered decisions, patient's preferences, and complete patient profiles should be provided in every patient presentation.

Although this is the first study of MDT treatment implementations in patients with complex extremity defects, the main limitation of this study stem from its retrospective observational study designspecifically, the potential for confounders, lack of clinical detail, and selection bias. Furthermore, the small sample size may limit the generalizability of our findings. Therefore, further prospective studies are 
necessary to analyze if the quality of care improves with MDT extremity boards in this challenging patient population.

\section{Conclusions}

MDT meetings for patients with complex extremity defects offer a platform to formulate individual treatment plans and to avoid limb amputation in the majority of cases. Patient preferences and patientrelated factors are crucial and must be considered to successfully implement the reached MDT meeting decision.

\section{Abbreviations}

MDT multidisciplinary team meeting

SD standard deviation

$\mathrm{Cl}$ confidence interval

\section{Declarations}

Ethics approval and consent to participate: The local ethics committee of Rhineland palatinate (no. 202015004) Mainz Germany approved the retrospective study, which was designed in accordance with the ethical standards laid down in the Declaration of Helsinki and its later amendments. Since the study protocol included only a retrospective, epidemiologic evaluation of anonymized and routine patient demographics, procedural and outcome specific parameters informed consent was waived by the local ethics commitee of Rhineland palatinate.

Consent for publication: not applicable since no identifying pictures are included

Availability of data and materials: All data is contained within the manuscript and other patient's details analysed during the current study available from the corresponding author on reasonable request.

Competing interests: not applicable

Funding: no funding was received, not applicable

Authors' contributions: Study conception and design: DKF, SF and UK. Acquisition of the data: JW, CH GR and PG; Analysis and interpretation of the data: DKF, SF and UK; Drafting of the manuscript: DKF and SF; Critical revision: UK, CH, JW, GR and PG. All authors approved the final version of the manuscript.

Acknowledgements: We thank Spyridoula Maraka, Assistant Professor of Medicine, University of Arkansas for Medical Sciences, for her assistance in revising the manuscript.

Authors' information: not applicable 


\section{References}

1. Van Den Heuvel SCM, Winters HAH, Ultee KH, Zijlstra-Koenrades N, Sakkers RJB: Combined massive allograft and intramedullary vascularized fibula transfer: the Capanna technique for treatment of congenital pseudarthrosis of the tibia. Acta Orthop 2020:1-6.

2. Morelli I, Drago L, George DA, Gallazzi E, Scarponi S, Romano CL: Masquelet technique: myth or reality? A systematic review and meta-analysis. Injury 2016, 47 Suppl 6:S68-S76.

3. Luo W, Huang L, Liu H, Qu W, Zhao X, Wang C, Li C, Yu T, Han Q, Wang J et al: Customized Knee Prosthesis in Treatment of Giant Cell Tumors of the Proximal Tibia: Application of 3-Dimensional Printing Technology in Surgical Design. Med Sci Monit 2017, 23:1691-1700.

4. Fischer S, Soimaru S, Hirsch T, Kueckelhaus M, Seitz C, Lehnhardt M, Goertz O, Steinau HU, Daigeler A: Local tendon transfer for knee extensor mechanism reconstruction after soft tissue sarcoma resection. J Plast Reconstr Aesthet Surg 2015, 68(5):729-735.

5. Fischer S, Diehm Y, Hirche C, Kremer T, Daigeler A, Kneser U, Hernekamp JF: Comparison of subversus suprafascially raised anterolateral thigh free flaps with regard to donor-site morbidity, function and aesthetics. Microsurgery 2018, 38(5):444-449.

6. Bigdeli AK, Gazyakan E, Schmidt VJ, Bauer C, Germann G, Radu CA, Kneser U, Hirche C: Long-Term Outcome after Successful Lower Extremity Free Flap Salvage. J Reconstr Microsurg 2019, 35(4):263-269.

7. Kallio M, Vikatmaa P, Kantonen I, Lepantalo M, Venermo M, Tukiainen E: Strategies for free flap transfer and revascularisation with long-term outcome in the treatment of large diabetic foot lesions. Eur J Vasc Endovasc Surg 2015, 50(2):223-230.

8. Reiter G, Thomas B, Kuhner C, Hundeshagen G, Weil F, Wittenberg G, Kloos S, Grutzner PA, Kneser U: [Reconstruction of lower limbs in old age-an interdisciplinary approach: Strategies for trauma surgery, vascular surgery and plastic surgery]. Chirurg 2019, 90(10):806-815.

9. Soukup T, Petrides KV, Lamb BW, Sarkar S, Arora S, Shah S, Darzi A, Green JS, Sevdalis N: The anatomy of clinical decision-making in multidisciplinary cancer meetings: A cross-sectional observational study of teams in a natural context. Medicine (Baltimore) 2016, 95(24):e3885.

10. Schmidt VJ, Hirsch T, Osinga R, Kotsougiani D, Schaefer DJ, Pierer G, Kolbenschlag J, Hulsemann W, Kneser U, Lehnhardt M: [The interdisciplinary Microsurgeon - Results of the Consensus Workshop of the German Speaking Society for Microsurgery of Peripheral Nerves and Vessels]. Handchir Mikrochir Plast Chir 2019, 51(4):295-301.

11. Samarasinghe A, Chan A, Hastrich D, Martin R, Gan A, Abdulaziz F, Latham M, Zissiadis Y, Taylor M, Willsher $\mathrm{P}$ : Compliance with multidisciplinary team meeting management recommendations. Asia Pac J Clin Oncol 2019, 15(6):337-342.

12. Pillay B, Wootten AC, Crowe H, Corcoran N, Tran B, Bowden P, Crowe J, Costello AJ: The impact of multidisciplinary team meetings on patient assessment, management and outcomes in oncology settings: A systematic review of the literature. Cancer Treat Rev 2016, 42:56-72. 
13. Jalil R, Ahmed M, Green JS, Sevdalis N: Factors that can make an impact on decision-making and decision implementation in cancer multidisciplinary teams: an interview study of the provider perspective. Int J Surg 2013, 11(5):389-394.

14. Butow P, Harrison JD, Choy ET, Young JM, Spillane A, Evans A: Health professional and consumer views on involving breast cancer patients in the multidisciplinary discussion of their disease and treatment plan. Cancer 2007, 110(9):1937-1944.

15. Choy ET, Chiu A, Butow P, Young J, Spillane A: A pilot study to evaluate the impact of involving breast cancer patients in the multidisciplinary discussion of their disease and treatment plan. Breast 2007, 16(2):178-189.

16. Hamilton DW, Heaven B, Thomson RG, Wilson JA, Exley C: Multidisciplinary team decision-making in cancer and the absent patient: a qualitative study. BMJ Open 2016, 6(7):e012559.

17. Lamb BW, Sevdalis N, Vincent C, Green JS: Development and evaluation of a checklist to support decision making in cancer multidisciplinary team meetings: MDT-QulC. Ann Surg Oncol 2012, 19(6):1759-1765.

18. Black CK, Ormiston LD, Fan KL, Kotha VS, Attinger C, Evans KK: Amputations versus Salvage: Reconciling the Differences. J Reconstr Microsurg 2019.

19. Wukich DK, Raspovic KM: What Role Does Function Play in Deciding on Limb Salvage versus Amputation in Patients With Diabetes? Plast Reconstr Surg 2016, 138(3 Suppl):188S-195S.

20. Endean ED, Mallon LI, Minion DJ, Kwolek CJ, Schwarcz TH: Telemedicine in vascular surgery: does it work? Am Surg 2001, 67(4):334-340; discussion 340 - 331.

\section{Figures}




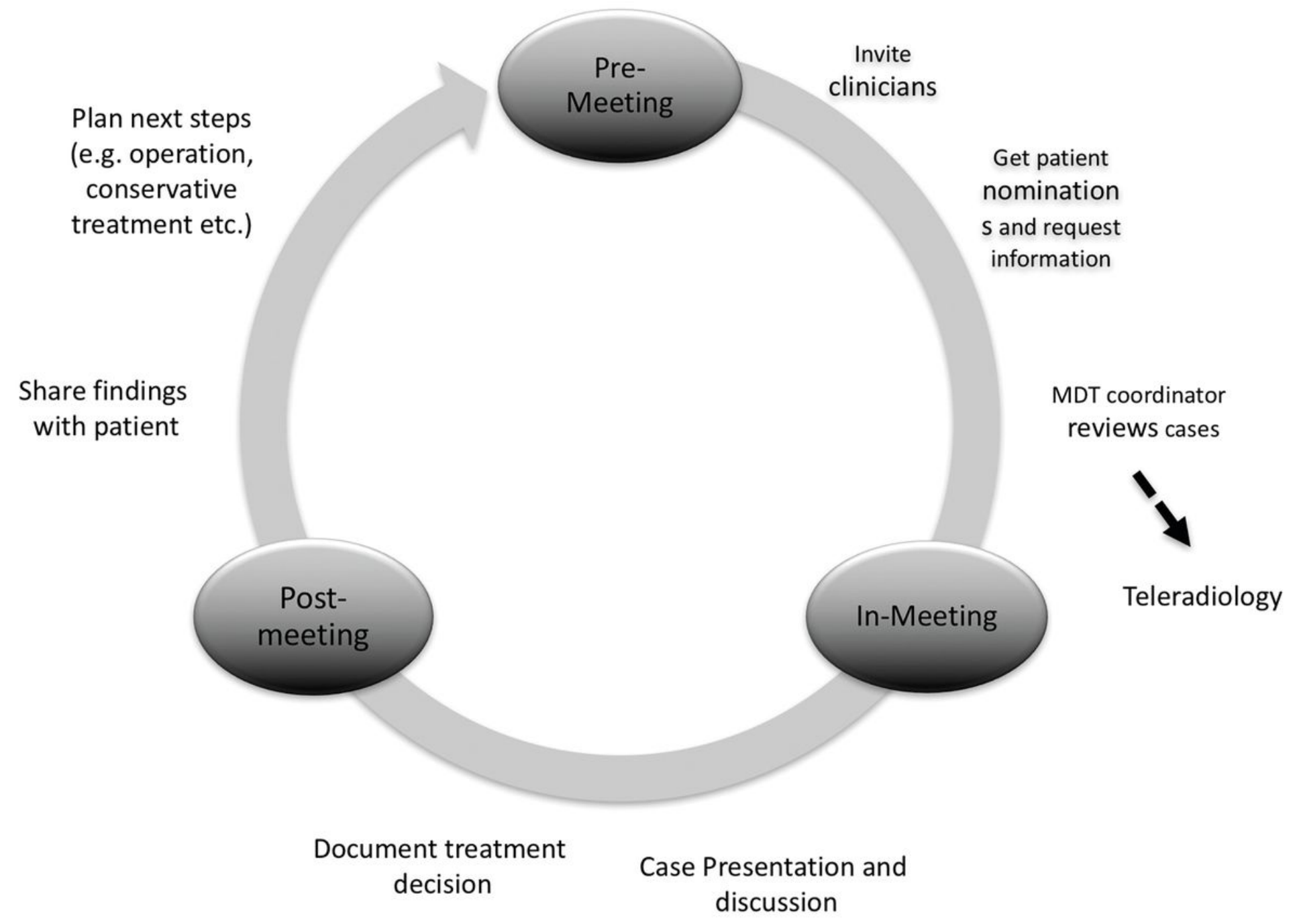

\section{Figure 1}

MDT meeting for complex extremity defects. Schematic drawing of the workflow of an MDT meeting for complex extremity defects 

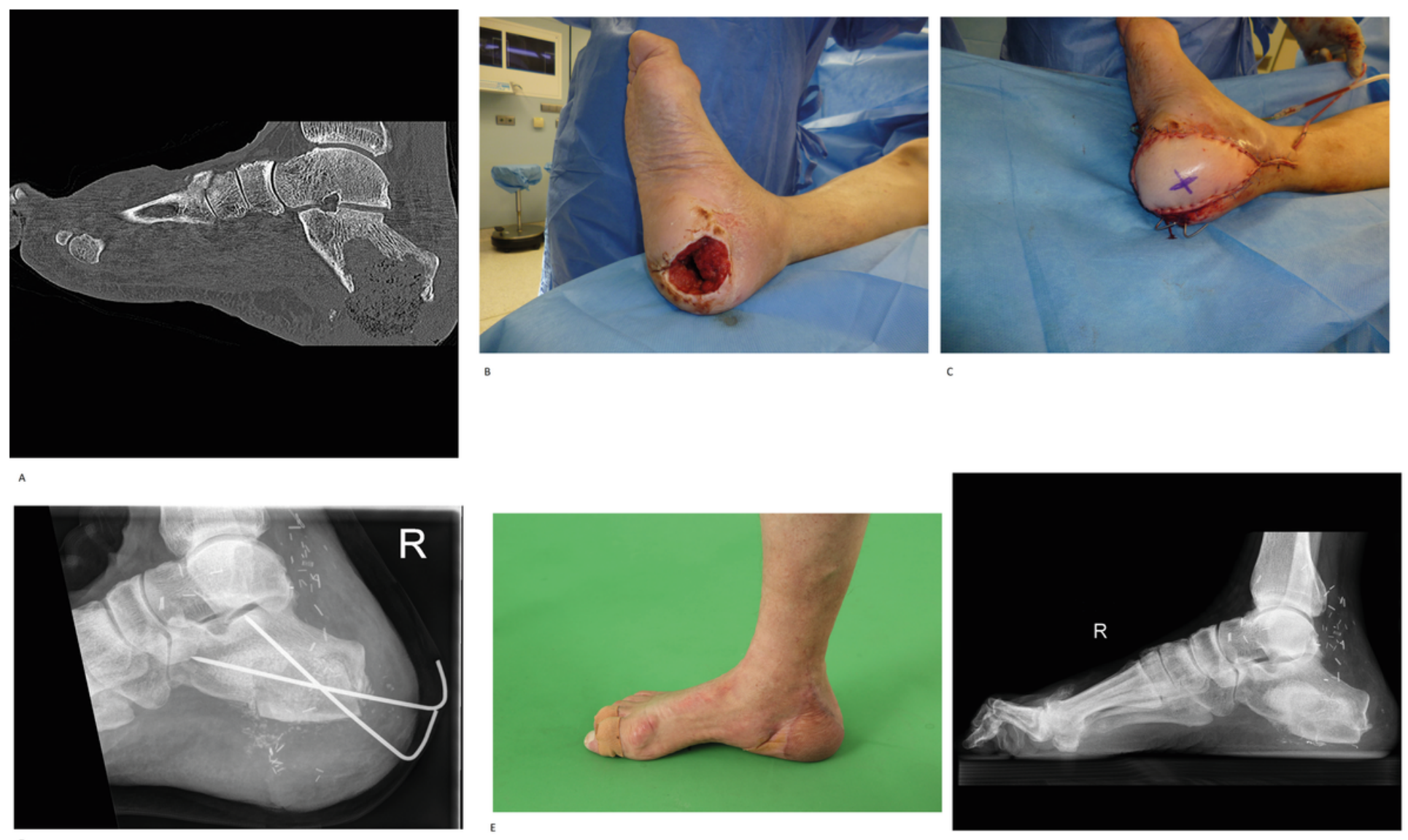

\section{Figure 2}

Limb-threatening chronic osteomyelitis of the calcaneus with subtotal resorption of the calcaneus and concomitant heel ulcers in a 66-year-old male patient. (a) Computed tomographic imaging of the right foot with subtotal bone resorption of the calcaneus due to chronic osteomyelitis. (b) Intraoperative lateral view of the right lower leg and foot with a major soft-tissue defect in the right weight-bearing heel before reconstruction. (c) Postoperative lateral view of the reconstructed heel. The bone defect has been reconstructed with a free vascularized iliac crest flap and the soft-tissue defect with a free groin flap. Inflap anastomoses were performed between the nutrient vessels of the iliac crest and groin flap and further anastomoses performed to the A. tibialis posterior. Furthermore, $15 \mathrm{cc}$ bioactive glass scaffold implantation was performed. (d) X-ray of the right foot21 days after internal fixation of the iliac crest bone segment by two k-wires. (e+f) Longterm follow-up after one year showing full weight-bearing and sufficient consolidated right calcaneus in the x-ray. 